\title{
Masculinidad y fútbol: cuestiones de género en una experiencia de rehabilitación psicosocial de hombres en el Distrito Federal, Brasil
}

\author{
Masculinity and soccer: gender issues in a psychosocial \\ rehabilitation experience with men in Brazil's Federal \\ District
}

${ }^{1}$ Psicólogo, Doctorando en Medicina Preventiva. Investigador, Departamento de Medicina Preventiva, Universidade de São Paulo, São Paulo, Brasil. $\triangle$ iD

${ }^{2}$ Médica, Doctora en Medicina. Profesora, Departamento de Medicina Preventiva, Faculdade de Medicina, Universidade de São Paulo. Investigadora $1 \mathrm{~A}$, Conselho Nacional de Desenvolvimento Científico e Tecnológico $(\mathrm{CNPq})$, Brasil. $\square$ (iD)
Fernando Pessoa de Albuquerque', Lilia Blima Schraiber ${ }^{2}$

RESUMEN Se aborda la participación de hombres en talleres de fútbol realizados en servicios de salud mental, deporte que es considerado una práctica importante en los procesos de sociabilidad de los hombres. Se realizó una investigación cualitativa en dos servicios de salud mental del Distrito Federal de Brasilia, entre agosto de 2017 y septiembre de 2018, con observación participante de las actividades habituales de los servicios y diez entrevistas semiestructuradas a usuarios varones, seleccionados durante las actividades observadas. Los hallazgos muestran la potencialidad del taller terapéutico de fútbol en la rehabilitación psicosocial de hombres con trastornos mentales, usuarios de los servicios de salud mental, a partir de la reinserción social y cultural en una actividad material y simbólicamente constructora de la masculinidad y de lo que representa ser hombre en Brasil. Estos pacientes, por ser portadores de sufrimientos mentales, suelen ser marginados y discriminados del modelo de masculinidad hegemónica, por lo cual, al viabilizar el ejercicio de una masculinidad posible, estos usuarios pueden expresar la forma de reconstruir su nueva identidad de ser hombre.

PALABRAS CLAVES Salud del Hombre; Masculinidades; Salud Mental; Centro de Atención Psicosocial; Brasil.

\begin{abstract}
This article presents a study of men's participation in soccer workshops at a mental health services facility (CAPS). The sport is considered a relevant practice in terms of men's sociability processes. Qualitative research was conducted at two CAPS facilities in Brasilia, Federal District from August 2017 to September 2018. Data were collected through observations of daily activities and with 10 semi-structured interviews with male participants who were selected during observations. The findings of this study demonstrate the potential of therapeutic soccer workshops for the psychosocial rehabilitation of men with mental disorders - the users of these mental health services - based on social and cultural re-insertion through an activity that materially and symbolically constructs masculinity and what it means to be a man in Brazil. As patients with mental disorders who are customarily marginalized from hegemonic masculinity, the users of CAPS services were able to access possible masculinities and reconstruct their new identities as men.

KEY WORDS Men's Health; Masculinities; Mental Health; Psychosocial Care Center; Brazil.
\end{abstract}




\section{INTRODUCCIÓN}

El presente estudio aborda la relación de hombres con diagnóstico de trastornos mentales con los talleres terapéuticos de los centros de atención psicosocial (CAPS) en la región circundante del Distrito Federal de Brasilia (Brasil), con énfasis en las prácticas de fútbol en los servicios de salud mental.

El acceso y la adhesión de los hombres a la atención en salud mental debe superar una serie de obstáculos relacionados con la construcción de la masculinidad y las normas requeridas para afirmarse en lo que es "ser un hombre". Los patrones de género hegemónicos producen referencias de comportamiento y desempeño social que llevan a los hombres a guardar silencio sobre sus problemas de salud, a menos que se los consideren serios. Algunos estudios señalan la dificultad de los hombres para expresar sentimientos y lidiar con las emociones, que causa un impacto negativo en su salud mental. Se podría decir que la cultura patriarcal y chauvinista estructura una relación compleja y comprometida en la forma en que los hombres manejan sus emociones. También se puede decir que los hombres silencian sus emociones y propia la subjetividad. Después de todo, los hombres ven la depresión como un signo de debilidad ${ }^{(1,2,3,4,5)}$.

Las vulnerabilidades descritas se exacerban debido a las dificultades de acceso a los servicios de salud que enfrentan los hombres. Los estudios indican que los hombres tienen menos consultas médicas por año en comparación con las mujeres ${ }^{(6)}$ y hacen menos uso de los servicios de cuidados progresivos tales como la atención primaria de salud ${ }^{(7)}$, ya que valoran subjetivamente menos los servicios de salud ${ }^{(8)}$, especialmente aquellos que se caracterizan por ofrecer cuidados prolongados, como los servicios de atención psicosocial.

Con relación a la atención en salud mental hay pocos estudios disponibles sobre el acceso de los hombres a servicios especializados de salud mental y, especialmente, sobre cómo se relacionan con ellos como usuarios. El estudio de Campos et al. ${ }^{(9)}$ muestra una demanda reducida de atención de salud mental por parte de los hombres. Sin embargo, los hombres presentan problemas de salud significativos que deben ser mejor observados, acogidos y atendidos por la Red de Atención Psicosocial.

Según Zanello et al. ${ }^{(10)}$, los servicios que componen la Red de Atención Psicosocial abordan muy poco las diferencias de género, y no desarrollan actividades específicas para hombres y/o mujeres. A pesar de que colocar la "enfermedad entre paréntesis" para poder acoger y cuidar la singularidad de cada sujeto $^{(11)}$ sea un principio de la reforma psiquiátrica, base conceptual de la Red de Atención Psicosocial, se observa que los servicios de salud mental enfocan sus acciones en la dimensión biopatológica del trastorno mental, reduciéndolo a la esfera biomédica y descuidando la vida social y, por lo tanto, aquellas cuestiones relacionadas con el género, así como con otras dimensiones de las desigualdades sociales, como la raza, la clase social y aspectos generacionales ${ }^{(12)}$. Un ejemplo de esto es la política de 2004 sobre la atención al consumo de alcohol, en la que no se mencionan acciones específicas sobre la población masculina y no se discute la relación entre el alcohol y los procesos de socialización masculina $^{(13)}$, a pesar de que este problema es mucho más frecuente en la población de sexo masculino.

Por lo tanto, se puede establecer la relevancia de investigar la rutina de los servicios de atención psicosocial y los talleres terapéuticos que se realizan como parte del tratamiento, para comprender cómo se pueden abordar las construcciones de género en la vida diaria de estos servicios, para abordar la influencia de las concepciones de masculinidad en la experiencia de los trastornos mentales y del comportamiento en el ámbito de los CAPS.

El Ministerio de Salud define el taller terapéutico en los CAPS como "Acciones desarrolladas colectivamente que exploran las potencialidades de las situaciones grupales [...] como un recurso para promover sociabilidad, intermediar las relaciones, manejar las dificultades relacionales, permitir 
la experiencia de construcción compartida, experimentar la pertenencia, intercambio de afecto, autoestima, autonomía y ejercicio de la ciudadanía"(14).

En última instancia, estos talleres terapéuticos tienen como finalidad aumentar la autonomía y promover la reintegración psicosocial de los usuarios ${ }^{(15)}$ e incluyen desde círculos de conversación, actividades artísticas, expresivas, musicales, producción artesanal y generación de ingresos, hasta prácticas corporales tales como el taller de futbol. Algunos talleres se llevan a cabo en espacios comunitarios, precisamente para fomentar la posibilidad de inclusión social y comunitaria de usuarios de servicios de salud mental ${ }^{(16)}$, que históricamente están estigmatizados y excluidos de la vida social[ ${ }^{(17)}$. Se observa que muchos CAPS desarrollan el taller de fútbol en espacios públicos, según lo informado por los pocos artículos que abordan el tema del fútbol dentro de los servicios de atención psicosocial ${ }^{(16,18,19,20)}$.

En este artículo, se destaca el taller de fútbol del CAPS porque este deporte tiene una representación muy relevante en los procesos de sociabilidad de los hombres, siendo uno de los elementos de construcción de la masculinidad en el contexto nacional, ya que "la práctica del fútbol es uno de los espacios de vivencia y formación de masculinidad hegemónica"(21). Además, esta actividad física compone una pedagogía de los cuerpos masculinos, que son educados y probados para lograr la "virilidad"(22).

Al definir el fútbol como un deporte que exige "resistencia varonil", los brasileños lo han transformado en una prueba de masculinidad. Los niños que no muestran talento ni gusto por el juego no pasan la prueba. [...] Del mismo modo, el acto de "hinchar" en un adulto muestra que tiene intereses masculinos. ${ }^{(22)}$

Las ideas de Souza ${ }^{(22)}$ estimularon esta investigación, con el propósito de profundizar el conocimiento sobre la relación de los hombres usuarios de servicios de salud mental con el taller terapéutico de fútbol, que reproduce uno de los principales modos de socialización de hombres y niños en Brasil. Además, buscamos comprender el papel de esta actividad en el proceso terapéutico de estos usuarios y la forma en que participan de los talleres propuestos por los servicios.

\section{MÉTODO}

Este estudio es parte de una investigación doctoral más amplia titulada "Sofrimento Mental e Gênero: o cuidado aos homens na Rede de Atenção Psicossocial", que investiga la interacción de los hombres que presentan demandas relacionadas con la salud mental con los servicios de la red de atención psicosocial del Distrito Federal en Brasilia, y cómo estos servicios de salud han tratado los motivos de consulta sobre el sufrimiento mental masculino, analizando esos motivos, las demandas y la atención ofrecida desde la perspectiva de género y de los estudios sobre la construcción social de las masculinidades. Para ello, se optó por una metodología cualitativa, ya que analizamos los aspectos subjetivos de la relación de los usuarios masculinos con el ejercicio de sus masculinidades y también con los servicios de atención psicosocial en los que fueron atendidos.

Se tomaron en consideración los principios de investigación cualitativa, considerados aquí como prácticas interpretativas que contemplan los significados que los sujetos atribuyen a los fenómenos y el conjunto de relaciones en las que se insertan ${ }^{(23,24)}$.

Las técnicas utilizadas para recopilar información fueron observación participante y entrevistas semiestructuradas a los usuarios masculinos que asistían a los servicios antes mencionados. La observación participante se realizó en el marco de las actividades diarias de estos servicios y de una semana típica de la rutina de cada punto de atención.

Las observación participante y las entrevistas semiestructuradas se basaron en guiones preparados sobre la base de los objetivos del proyecto de investigación y en diálogo con las preguntas planteadas en investigaciones 
recientes sobre el tema, como la producción de Zanello et al. ${ }^{(10,25)}$, Schraiber et al. ${ }^{(6)}$ y Figueiredo et al.(26), que también sirvieron de apoyo para las entrevistas y fueron utilizadas como preguntas claves, manteniendo el respeto por el flujo de ideas de los informantes, con énfasis en la percepción de los usuarios relacionada con sus relaciones con el servicio de salud mental.

Los servicios involucrados fueron dos CAPS en las afueras de Distrito Federal en Brasilia, que se encuentran en la región de Samambaia: un CAPS III 24 horas que brinda atención a adultos con trastornos mentales graves y persistentes, y un centro de atención psicosocial, alcohol y drogas (CAPS-AD) III 24 horas, que atiende a personas con trastornos por consumo de alcohol y otras drogas.

Las observaciones en los servicios de salud de la Red de Atención Psicosocial también sirvieron para identificar posibles sujetos para ser entrevistados, a quienes se contactó a partir de las actividades de atención observadas. En total, se entrevistaron diez usuarios, todos pacientes regulares de los dos CAPS incluidos en la investigación. La observación participante y las entrevistas semiestructuradas se realizaron entre agosto de 2017 y septiembre de 2018.

Los datos se analizaron a partir de las particularidades de su construcción, su relación con los contextos en los que se generaron y desde el punto de vista del informante, considerando a cada uno como un individuo que representa la cultura a la que pertenece, y que adhiere al imaginario social construido, aunque tenga representaciones particulares de esa cultura ${ }^{(24)}$.

Se realizaron reiteradas lecturas del material transcrito de las entrevistas y el diario de campo producido durante las observaciones, para permitirle al investigador captar el contenido del texto, en el proceso llamado impregnación ${ }^{(27)}$.

Se elaboró una primera categorización de temas, siguiendo los ejes del guion de la observación y el guion de la entrevista, basados en nuestro marco teórico. Del material empírico surgieron otros contenidos subyacentes, lo que nos llevó a abrir temas en categorías internas más limitadas, pero no menos relevantes.

Intentamos analizar el material recopilado, las diez entrevistas transcritas y los diarios de campo de cada servicio, a partir de la comprensión y contextualización de los significados atribuidos por los entrevistados a los temas tratados en las entrevistas, que se registraron y luego se transcribieron en su totalidad.

Para este estudio extrajimos del análisis los contenidos relacionados con la participación en el taller terapéutico de fútbol en ambos CAPS, utilizando como fuentes las entrevistas y diarios de campo. Todos los pacientes entrevistados habían asistido al taller de fútbol al menos una vez, y cinco de ellos fueron invitados a la entrevista durante esa actividad. Los otros entrevistados fueron contactados en grupos terapéuticos de rutina.

El uso de la observación participante permitió el análisis de cómo ocupan los espacios y actividades de estos servicios, con una mirada en profundidad a la práctica del fútbol como taller terapéutico en los CAPS. Vale la pena mencionar que esta técnica nos permite comprender el conjunto de entendimientos y significados compartidos en una práctica colectiva $^{(28)}$, mientras que las entrevistas permiten la comprensión de los significados atribuidos por los hombres a la práctica del fútbol en el servicio y su relación con el ejercicio de masculinidad y sociabilidad entre hombres. En el análisis de las entrevistas, se decidió identificar núcleos de significado relacionados con la práctica del fútbol y su relación con el ejercicio de la masculinidad y la sociabilidad entre los hombres, utilizando como base el marco teórico de los estudios de género.

Posteriormente, las ideas explícitas e implícitas en el texto se problematizaron con informes tomados del diario de campo, de observaciones y de entrevistas, con significados socioculturales más amplios. Finalmente, se propuso una primera síntesis interpretativa, que buscaba articular entre los objetivos del estudio, las bases teóricas adoptadas y los datos empíricos.

El proyecto de investigación, junto con los términos de consentimiento informado 
para observación participante, entrevista con profesionales de la salud y usuarios, fueron aprobados por el Comité de Ética de Investigación de la Facultad de Medicina de la Universidade de São Paulo (Código No. 1.913.518) y por el comité de ética en investigación de la Fundação Ensino e Pesquisa em Ciências da Saúde de la Secretaría de Salud del Distrito Federal (Código No. 2.137.522).

\section{RESULTADOS}

\section{Perfil de los entrevistados y de las actividades observadas}

Para contextualizar las declaraciones tomadas de cada entrevista, se decidió relatar brevemente el historial de los problemas de salud mental experimentados por cada entrevistado. Las verdaderas identidades se mantuvieron en secreto y se reemplazaron por nombres relacionados con personajes de canciones de grupos de rock de Brasilia, ya que todos los entrevistados son hombres que viven las diferentes realidades del Distrito Federal. La presentación de los entrevistados está en la Tabla 1.

Con respecto a los grupos de edad, tres entrevistados tenían entre 20 y 30 años, seis entre 30 y 50 años y solo uno más de 60 años. Ocho hombres se identificaron como negros, siete de piel parda y solo dos se autoidentificaron como blancos. Con respecto a la orientación sexual, los diez se declararon heterosexuales, sin informes de relaciones homosexuales durante las declaraciones. En cuanto al estado civil, la mitad de los entrevistados eran solteros, seguidos de tres casados y dos separados. Es importante destacar que siete de ellos no tenían una relación estable durante el período de investigación, en consonancia con aquellos estudios que indican que los hombres solteros tienen más trastornos mentales que los casados ${ }^{(29)}$.

En referencia a la situación laboral, siete entrevistados no tenían ingresos propios, de los cuales tres estaban en busca de un certificado médico para obtener la jubilación por discapacidad. De los tres que recibieron algún tipo de ingreso, solo uno trabajaba, otro era un funcionario público retirado y, el tercero, un guardia de seguridad que se retiró a través del Instituto Nacional de Seguro Social debido a un tratamiento mental, en este caso, por un reciente brote psicótico.

Solo tres entrevistados eran del Distrito Federal, tres provenían de Bahía, tres de Minas Gerais y solo uno de Goiás. Este último era el único que hacía menos de 7 años que había Ilegado al Distrito Federal, todos los demás migrantes se habían mudado al Distrito Federal entre 7 y 49 años atrás.

Con respecto al servicio utilizado, siete eran pacientes regulares del CAPS III y tres del CAPS-AD. Esta diferencia se debía al hecho de que el campo en el CAPS-AD era más pequeño que en el CAPS III. Con respecto al perfil de los entrevistados por tipo de servicio, se observó que existían pocas diferencias con relación a la raza/color, el estado civil y la educación. El CAPS III mostraba un mayor número de pacientes adultos jóvenes, que habían desarrollado trastornos psicóticos durante la adolescencia, mientras que los usuarios de CAPS-AD entrevistados tenían entre 30 y 50 años.

La principal diferencia entre los dos servicios se debe a los tipos de motivos de consulta y diagnósticos presentados por cada entrevistado. En el CAPS III, se observó un mayor número de diagnósticos de esquizofrenia o motivos de consulta relacionadas con síntomas psicóticos, y seis de los siete entrevistados informaron síntomas psicóticos como alucinaciones o delirios persecutorios. Mientras que en el CAPS-AD el diagnóstico de dependencia química se observó en todos los entrevistados, y solo uno usó alcohol exclusivamente. Los otros dos entrevistados registraban abuso de alcohol y crack.

Los entrevistados del CAPS III habían usado regularmente este servicio desde una semana a dos años, y solo dos de los siete entrevistados del CAPS III habían recibido tratamiento en este servicio durante menos de un año. Se observó que la duración de la estadía en el tratamiento era mayor entre los usuarios del CAPS III, ya que entre los pacientes del CAPS-AD, dos de ellos habían asistido 
Tabla 1. Caracterización de los usuarios entrevistados. Brasilia, 2017-2018.

\begin{tabular}{|c|c|c|c|c|c|c|c|c|c|}
\hline Servicio & Nombre & Edad & $\begin{array}{l}\text { Lugar de } \\
\text { nacimiento } \\
\text { (años y lugar de } \\
\text { residencia actual) }\end{array}$ & Raza & $\begin{array}{l}\text { Situación } \\
\text { Conyugal }\end{array}$ & Profesión & Escolaridad & $\begin{array}{l}\text { Motivo de } \\
\text { consulta/ } \\
\text { Diagnóstico } \\
\text { principal }\end{array}$ & Breve relato del caso \\
\hline CAPS III & Pablo & 47 & $\begin{array}{l}\text { Bahía } \\
\text { (hace } 24 \text { años en } \\
\text { Distrito Federal) }\end{array}$ & Pardo & Casado & $\begin{array}{l}\text { Vigilante (licencia } \\
\text { por motivo de } \\
\text { salud) }\end{array}$ & $\begin{array}{l}\text { Educación } \\
\text { superior } \\
\text { completa }\end{array}$ & $\begin{array}{l}\text { Brote psicótico } \\
\text { reciente }\end{array}$ & $\begin{array}{l}\text { Trabaja como vigilante en un servicio de salud, llegó al CAPS III, para internación } \\
\text { integral después de la hospitalización en el Hospital Psiquiátrico debido a un } \\
\text { brote psicótico que tuvo durante las horas de trabajo, en el que disparó cuando } \\
\text { se reunió con un sindicalista a quien acusó de perseguirlo. }\end{array}$ \\
\hline CAPS III & Maurício & 22 & $\begin{array}{l}\text { Piauí } \\
\text { (hace } 7 \text { años en } \\
\text { Distrito Federal) }\end{array}$ & Blanco & Soltero & Desempleado & $\begin{array}{l}\text { Educación } \\
\text { media } \\
\text { completa }\end{array}$ & Esquizofrenia & $\begin{array}{l}\text { Es un joven de Piauí, llegó a estar en situación de calle en su ciudad natal de } \\
\text { Piauí, hasta que su tío lo trajo a Brasilia. Se relatan síntomas de esquizofrenia } \\
\text { desde la edad de } 14 \text { años, y desde las primeras convulsiones su tío lo llevó a } \\
\text { recibir tratamiento en el Hospital Universitario de Brasilia y luego fue derivado } \\
\text { al CAPS. }\end{array}$ \\
\hline CAPS III & Luís & 23 & $\begin{array}{l}\text { Minas Gerais } \\
\text { (hace } 18 \text { años en } \\
\text { Distrito Federal) }\end{array}$ & Pardo & Soltero & Desempleado & $\begin{array}{l}\text { Educación } \\
\text { primaria } \\
\text { incompleta }\end{array}$ & Autismo & $\begin{array}{l}\text { Le diagnosticaron autismo desde que tenía seis años y estaba en tratamiento } \\
\text { en un servicio especializado en orientación médica y psicopedagógica, pero } \\
\text { también presentó síntomas psicóticos, según los registros médicos consultados. } \\
\text { Llegó al CAPS por un servicio de referencia de psicopedagogía. }\end{array}$ \\
\hline CAPS III & André & 38 & Distrito Federal & Pardo & Soltero & $\begin{array}{l}\text { Supermercado } \\
\text { Desempleado }\end{array}$ & $\begin{array}{l}\text { Educación } \\
\text { media } \\
\text { completa }\end{array}$ & $\begin{array}{l}\text { Sin diagnóstico/ } \\
\text { Presentó brote } \\
\text { psicótico }\end{array}$ & $\begin{array}{l}\text { Informó haber tenido un brote psicótico hace un año durante las horas de } \\
\text { trabajo. No informa qué lo llevó a ser admitido en el Hospital Psiquiátrico por un } \\
\text { período tampoco especificado. Sin embargo, él cree que, si toma el medicamento } \\
\text { correctamente durante dos años, será tratado y puede volver a trabajar. Llegó al } \\
\text { CAPS III derivado desde el Hospital Psiquiátrico. }\end{array}$ \\
\hline CAPS III & Johnny & 35 & $\begin{array}{l}\text { Minas Gerais } \\
\text { (hace } 25 \text { años en } \\
\text { Distrito Federal) }\end{array}$ & Negro & Separado & $\begin{array}{l}\text { Profesor de jiu- } \\
\text { jitsu Desempleado }\end{array}$ & $\begin{array}{l}\text { Educación } \\
\text { primaria } \\
\text { incompleta }\end{array}$ & Esquizofrenia & $\begin{array}{l}\text { Informó que no podía dormir debido a crisis de agresión, automutilación y } \\
\text { alucinaciones de ideas suicidas, que se han intensificado desde que se separó } \\
\text { hace tres años. Una vez vivió en situación de calle, pero actualmente vive con } \\
\text { su madre. Después de una serie de hospitalizaciones en el Hospital Psiquiátrico, } \\
\text { primero fue derivado al CAPS-AD, pero dijo que hubo un error, corregido cuando } \\
\text { fue derivado al CAPS III, donde recibió tratamiento durante cinco meses. Desde } \\
\text { que fue hospitalizado, interrumpió sus clases de jiu-jitsu. }\end{array}$ \\
\hline CAPS III & Oscar & 69 & $\begin{array}{l}\text { Bahía } \\
\text { (hace } 49 \text { años en } \\
\text { Distrito Federal) }\end{array}$ & Pardo & Casado & $\begin{array}{l}\text { Trabajador } \\
\text { jubilado }\end{array}$ & $\begin{array}{l}\text { Educación } \\
\text { primaria } \\
\text { incompleta }\end{array}$ & Insomnio & $\begin{array}{l}\text { El único entrevistado adulto mayor fue seleccionado después de que en el grupo } \\
\text { de terapia comunitaria planteara preguntas interesantes sobre las relaciones de } \\
\text { los hombres con el alcohol y la interferencia de sustancias en la vida cotidiana } \\
\text { de las familias. Oscar es un empleado público jubilado e informa que sufre de } \\
\text { insomnio desde 2003, y llegó al CAPS solo después de someterse a tratamiento } \\
\text { en el Hospital Samambaia, donde no se sintió bienvenido. "En el CAPS tenemos } \\
\text { padrinos" }\end{array}$ \\
\hline CAPS III & Eduardo & 23 & Distrito Federal & Pardo & Soltero & Desempleado & $\begin{array}{l}\text { Educación } \\
\text { media } \\
\text { incompleta }\end{array}$ & Esquizofrenia & $\begin{array}{l}\text { Comenzó el tratamiento en este CAPS hace tres meses, y fue derivado por el } \\
\text { Instituto de Salud Mental del Distrito Federal, donde asistió a una serie de } \\
\text { actividades artísticas y de salud mental, que extraña y se pregunta por qué no lo } \\
\text { mantuvieron en el Instituto de Salud Mental. Es joven y vive con sus padres y su } \\
\text { hermana. Dijo que nunca estuvo de novio. Comenzó el tratamiento en el Instituto } \\
\text { de Salud Mental, después de haber tenido un brote con síntomas psicóticos, que } \\
\text { lo llevó a ingresar al hospital psiquiátrico del Distrito Federal y posteriormente } \\
\text { ser derivado al CAPS III. }\end{array}$ \\
\hline CAPS AD & Vidal & 30 & Distrito Federal & Pardo & Soltero & Desempleado & $\begin{array}{l}\text { Educación } \\
\text { primaria } \\
\text { completa }\end{array}$ & $\begin{array}{l}\text { Uso de alcohol y } \\
\text { otras drogas }\end{array}$ & $\begin{array}{l}\text { Vidal ha estado bajo tratamiento en el CAPS-AD durante dos años y medio } \\
\text { debido al abuso de alcohol y cocaína. Fue llevado por su padre, quien fue enviado } \\
\text { por un diputado del distrito a buscar el servicio. Actualmente es representante } \\
\text { de los pacientes del CAPS y desea establecer una asociación de usuarios. } \\
\text { Sin embargo, Vidal dijo que bebió y usó cocaína para aliviar su enojo con los } \\
\text { parientes paternos que lo acusaron de no ser un hijo legítimo porque tenía el } \\
\text { color de la piel más oscuro que su padre. Informó que los miembros de la familia } \\
\text { hicieron bromas racistas, diciendo que no se relacionaban con el "negro". Vidal } \\
\text { también dijo que solo alivió su enojo después de hacerse una prueba de ADN, lo } \\
\text { que demostró que era hijo legítimo. Este examen fue sugerido por un profesional } \\
\text { del CAPS. }\end{array}$ \\
\hline CAPS AD & João & 45 & $\begin{array}{l}\text { Goiás } \\
\text { (hace } 1 \text { año en } \\
\text { Distrito Federal) }\end{array}$ & Blanco & Separado & $\begin{array}{l}\text { Herrero } \\
\text { Desempleado }\end{array}$ & $\begin{array}{l}\text { Educación } \\
\text { media } \\
\text { completa }\end{array}$ & $\begin{array}{l}\text { Uso perjudicial de } \\
\text { alcohol }\end{array}$ & $\begin{array}{l}\text { João ha estado en Brasilia durante un año, viviendo con su hermana y recibiendo } \\
\text { tratamiento en el CAPS-AD durante tres meses. En el momento de la entrevista, } \\
\text { estaba en cuidado integral después de una recaída. Está recién separado. Él } \\
\text { dice que comenzó a beber demasiado después de perder a su hijo de } 17 \text { años, } \\
\text { asesinado por su participación en el tráfico de drogas. La familia de su esposa lo } \\
\text { acusa de ser culpable de la pérdida del joven por haberlo “abandonado". Lloraba } \\
\text { mucho cuando hablaba de su hijo. Estaba en consulta por un problema con sus } \\
\text { ojos. Al perder a su hijo, el médico que lo acompaña lo remitió al CAPS. Además, } \\
\text { le da vergüenza estar desempleado y no ser incapaz de mantener a su familia. }\end{array}$ \\
\hline CAPS AD & Jeremías & 38 & $\begin{array}{l}\text { Minas Gerais } \\
\text { (hace } 11 \text { años en } \\
\text { Distrito Federal) }\end{array}$ & Pardo & Casado & $\begin{array}{l}\text { Auxiliar de } \\
\text { limpieza }\end{array}$ & $\begin{array}{l}\text { Educación } \\
\text { primaria } \\
\text { incompleta }\end{array}$ & $\begin{array}{l}\text { Uso perjudicial de } \\
\text { alcohol y crack }\end{array}$ & $\begin{array}{l}\text { Jeremías estaba con cuidados integrales en el CAPS-AD. Dijo que } \\
\text { acudió al servicio por su cuenta, ya que en otras ocasiones fue } \\
\text { admitido en comunidades terapéuticas religiosas por insistencia de } \\
\text { miembros de la familia, pero fue la esposa quien le contó sobre la } \\
\text { existencia del CAPS-AD. Informó que tuvo problemas con el alcohol } \\
\text { hacía dos años, después de que tuvo su tercer hijo y no podía mantener } \\
\text { la casa. También declaró que comenzó a "deshacerse" de su esposa } \\
\text { mientras bebía. Además, dijo que ya había vivido en situación de } \\
\text { indigencia y también que había consumido crack. }\end{array}$ \\
\hline
\end{tabular}


al servicio durante menos de 3 meses y solo uno había sido un paciente regular durante dos años y medio.

Durante las entrevistas, se les preguntó cuánto tiempo habían vivido con estos problemas de salud mental que los llevaron a ser pacientes del CAPS. Solo uno de ellos (Pablo) declaró que tuvo su primera hospitalización psiquiátrica y, en consecuencia, había sido derivado al CAPS tres semanas antes de la entrevista. Sin embargo, todos los demás entrevistados habían vivido con estos problemas de salud mental durante más tiempo. Los entrevistados dijeron que habían vivido con estos problemas de salud mental durante aproximadamente seis años, cuatro de ellos informaron que tuvieron sus primeros síntomas hacía dos o tres años, y otros tres entrevistados informaron que habían tenido estos síntomas durante más de diez años.

Todos los entrevistados eran pacientes regulares y frecuentes de los servicios. En el CAPS III, solo uno tenía atención integral, internado en la unidad, y los otros seis usaban el servicio de dos a cuatro veces por semana participando en talleres grupales temáticos, especialmente, en la actividad de fútbol que era coordinada por un farmacéutico. El servicio no contaba con un profesional de educación física, pero este taller de fútbol tenía una propuesta recreativa que no requería la presencia de un especialista para realizarlo.

A su vez, en el CAPS-AD, dos entrevistados tenían atención integral y uno usaba el servicio todos los días hábiles. Todos participaban en un grupo terapéutico dirigido por un psicólogo y un enfermero, y parecían estar vinculados a los servicios, manteniendo relaciones regulares y afectivas tanto con profesionales como con otros usuarios.

\section{Hombres, masculinidades y uso de servicios de salud mental}

En la investigación realizada se observó la dificultad de incorporar a los hombres a espacios de atención de la salud típicamente ocupados por mujeres, porque no se sienten cómodos hablando de sí mismos con personas del sexo opuesto. Los estudios sobre la participación de los hombres en las actividades realizadas por los equipos de atención primaria corroboraron estos mismos obstáculos ${ }^{(7,8,30)}$. Por otra parte, las mujeres están más acostumbradas a tratar con las actividades que ofrecen los servicios de salud; cuando abordan cuestiones relacionadas con los sentimientos y las emociones, tienen un vocabulario mayor en relación con el universo psíquico y se sienten más cómodas hablando de sí mismas ${ }^{(2)}$. Este contexto dificulta que los hombres sean incluidos en las actividades grupales, considerando además que el modo de subjetivación masculina requiere callar las emociones, los defectos y las debilidades experimentadas ${ }^{(31)}$.

En el estudio de Campos et al.(9), los autores atribuyen la menor demanda de atención de los hombres en los servicios de salud mental a los patrones de masculinidad hegemónica, relacionados con un menor "autocuidado" y el "miedo a descubrir algo malo" sobre su salud mental"(9).

A pesar de esta conformación, se destaca la capacidad de estos servicios de establecer espacios de convivencia y vinculación entre usuarios en el CAPS III y el CAPS-AD. Durante las entrevistas, hubo muchos informes de usuarios que enfatizaban la calidad del vínculo entre los trabajadores del servicio y los pacientes. Además, la mayoría de los pacientes declararon que este era un lugar en el que se sentían más cómodos, donde sufrían menos discriminación que en otros espacios públicos y que realizaban algunas actividades dentro del servicio, que no harían en otro lugar. Por ejemplo, Eduardo, quien participaba en la actividad de fútbol en el CAPS III, informó que se sentía mejor allí porque era más parecido a los muchachos, en comparación con los muchachos de la viIla olímpica, donde también jugaba al fútbol esporádicamente.

La diferencia es que allá está la "society", hay personas normales... y aquí hay más colegas, más amigos ¿no? Me siento como un pez fuera del agua... (Eduardo) 
Aquí cabe retomar el tema de la inclusión/exclusión de pacientes estigmatizados por trastornos mentales que son discriminados y excluidos por ser considerados "locos". Se observa que la convivencia ocurre entre quienes fueron estigmatizados y excluidos, con una dificultad de reintegración social ${ }^{(32)}$.

En este caso, el CAPS tiene una función muy delicada; por un lado, incluye a estas personas en una red de relaciones que colabora con su tratamiento, pero a menudo se convierte en el único o uno de los pocos espacios donde viven estos pacientes, con el peligro de no colaborar en el fortalecimiento de los lazos familiares y comunitarios, y "enCAPSular" y aislarlos del exterior. Sin embargo, a partir de los datos recogidos en las observaciones y entrevistas de esta investigación, destacamos el papel positivo de estos servicios en el restablecimiento de lazos de confianza y sociabilidad, a través de las relaciones entre los usuarios. Los pacientes también enfatizan la calidad de las relaciones que los profesionales tienen con ellos, lo que parece ser un aspecto positivo del tratamiento. Esta relación de confianza parece disminuir la situación de impotencia en la que se encuentran los sujetos con sufrimiento mental.

Sin embargo, en las entrevistas se identificaron espacios en los que los hombres se sentían más o menos cómodos, como en el caso de Johnny, que se sintió avergonzado de verse a sí mismo como el único hombre en la reunión de padres de su hijo, sintiéndose desplazado en un entorno tradicionalmente ocupado por mujeres. La misma sensación de estar fuera de lugar se produjo en los grupos terapéuticos mixtos del CAPS III - Adulto, donde Johnny se sentía incómodo de estar.

\section{La práctica del fútbol en la rehabilitación psicosocial y las posibilidades de ejercicio de las masculinidades}

En los lugares típicos con más o menos presencia masculina, en los que los hombres se sienten más o menos cómodos, se notó en las entrevistas y en las transcripciones de las observaciones en el diario de campo, que la actividad de jugar al fútbol es muy importante para el proceso de socialización de estos pacientes masculinos en los CAPS. Después de todo, el taller de fútbol era el que tenía más hombres en el CAPS III y el único autogestionado por pacientes en el CAPS-AD. Se observó que la identificación de estos usuarios masculinos con los servicios de atención psicosocial se intensificaba durante el taller de fútbol en ambos servicios.

Según el diario de campo, el fútbol se realiza todos los miércoles por la mañana en el CAPS III, en una cancha pública a cinco minutos a pie, en una gran plaza con otras instalaciones, como un parque de skate. El taller es coordinado por un farmacéutico de 34 años. El diario de campo nos trae más información sobre el profesional:
En otro momento me contó que era un militar, por eso le da tanta importan- cia a la actividad física que, además de cuidar su cuerpo y su cabeza, nos deja más tranquilos. Dice que le encanta jugar a la pelota cualquier día de la semana. (Diario de campo, 8/8/2017, CAPS III, Samabaia)

El investigador participó en este taller siete veces durante el período de recolección de datos y señaló que los participantes en el grupo de fútbol son ligeramente más jóvenes que la mayoría de los pacientes de los CAPS, presentando edades comprendidas entre 20 y 25 años.

También se observó que estos pacientes jóvenes bromean entre ellos durante el juego, socializando de una manera muy relajada.

\section{El coordinador del taller le da un tono lúdico al juego, manteniendo el clima con poca competitividad, lo que parece redu- cir la ansiedad por el rendimiento de los pacientes, fomentando una mayor socia- bilidad y movimiento corporal. (Diario de campo, 16/8/2017, CAPS III, Samabaia)}

En el CAPS III, la mayoría de los entrevistados fueron invitados para ser entrevistados 
durante el taller de fútbol, que se llevaba a cabo en una cancha cercana. Los cinco pacientes que asistieron al taller informaron que se sintieron mejor y que el taller estaba colaborando en su tratamiento, como en el caso de Eduardo, quien afirmó que es necesario practicar actividad física para sentirse más hombre y que jugar a la pelota era bueno para su tratamiento. Luis y Mauricio también informaron que estaban mejorando, especialmente en relación con sus propios cuerpos, incluyendo disminución de temblores y menos impregnación, efectos secundarios del uso de medicamentos psiquiátricos.

Se decidió dar un mayor énfasis a este taller ya que, a partir de las entrevistas y el diario de campo, se evaluó que existía un gran potencial para la inclusión comunitaria y la promoción de la sociabilidad entre los pacientes, ya que esta práctica deportiva es parte del universo simbólico de subjetivación hegemónica y promueve un acercamiento con los pacientes al modelo tradicional de masculinidad. En este caso, dada la jerarquía y la competitividad que generalmente marcan la relación entre los hombres, está claro que llevar a cabo este taller entre pacientes los hace sentir más libres y más cómodos, como se puede ver en las siguientes declaraciones:

Eduardo: El fútbol, creo que es bueno... también lo practico en la Villa Olímpica... Entrevistador: ¿Cuál es la diferencia de jugar aquí y en la Villa Olímpica? Eduardo: La diferencia es que allá está la "society", están las personas normales ... y aquí hay más colegas, más amigos, ¿no? Allá (en la Villa Olímpica) me siento como un pez fuera del agua.

Entrevistador: ¿Por qué sentís que en la Villa Olímpica la gente te mira como alguien diferente?

Eduardo: El profesor sabe que soy... que tengo un problema... mi madre me recoge... cuando termina el fútbol él pregunta: ¿dónde está tu madre, no te viene a buscar? (Eduardo)

Con los muchachos juego porque tienen problemas como yo, con ellos no me da vergüenza. (Luís)
Se entiende que es una propuesta de sociabilidad limitada porque no permite la interactividad con personas de su propia comunidad, sino que tienen contacto solo entre los propios pacientes, reificando la típica segregación de los "enfermos mentales".

Sin embargo, esta misma situación también puede interpretarse desde la perspectiva del ejercicio de la masculinidad y su construcción sociohistórica, teniendo en cuenta que, como se mostró anteriormente, estos pacientes se sienten apartados de la modalidad socialmente requerida para ser hombres. Dada esta marginación de las formas de ser hombre, el fútbol puede verse en estos pacientes como una práctica afirmativa y en la afirmación del ejercicio de la masculinidad. Además, se puede observar el extracto del diario de campo, seguido de un breve análisis del observador participante:

El instructor propone que los pacientes dibujen un uniforme del equipo del CAPS, sugiere que pueden crear una insignia del equipo y acordar un partido con el equipo del otro CAPS de Samambaia, en este caso el CAPS-AD. Después de eso, les dijo a los muchachos que necesitaba mostrarles un video en su teléfono celular. Todos se acercaron y puso el himno del equipo de Flamengo. Algunos se quejan y se indignan. Uno dice, "acá somos del Curintia" [forma popular de Ilamar al equipo Corinthians] quejándose. Cinco participantes cantan junto a Diógenes. Me doy cuenta de que la identificación de estos muchachos con un hombre mayor, envueltos por la cultura del fútbol provoca una importante reinclusión social para ellos. Creo que, si estuvieran con muchachos que no tuvieran los mismos sufrimientos mentales, les sería más difícil ser aceptados para jugar en un equipo. Sigo pensando en la alegría de Maurício [paciente entrevistado] al hacer un gol, como un ejercicio de su masculinidad. (Diario de campo, 09/08/2017, CAPS III, Samambaia) 
Según Gastaldo ${ }^{(33)}$, la cultura del fútbol, incluido el juego y la hinchada por algún equipo, es parte de la actuación masculina del hombre brasileño, destacando el papel del juego en la construcción de la identidad:

\footnotetext{
Tradicionalmente, la participación en juegos, concursos y desafíos es un rasgo característico del género masculino en las culturas más diversas. De los grupos tribales de todo el mundo, en los grupos rurales y en nuestra sociedad urbana moderna, gran parte del significado articulado con "ser hombre" se relaciona con la aceptación de los desafíos planteados por otros hombres. ${ }^{(33)}$
}

Se observó que proponer estos desafíos en un entorno en el que los pacientes con sufrimiento mental se sienten más seguros conduce a una afirmación de la masculinidad y un sentimiento de prestigio e inclusión, lo que permite una sociabilidad positiva entre los hombres que, según algunos de los entrevistados, no tuvieron experiencias favorables e inclusivas durante su vida.

Es necesario profundizar el estudio del papel del fútbol en la rehabilitación psicosocial de hombres jóvenes con sufrimiento mental, teniendo en cuenta la importancia de la cultura del fútbol en la construcción de la masculinidad del hombre brasileño y discutir las posibilidades de utilizar los patrones de género como un rescate de su identidad ${ }^{(25)}$.

Por supuesto, existe el riesgo de una reafirmación de los patrones de identidad que limitan el ejercicio de la masculinidad a modelos hegemónicos, basados en la cultura de la virilidad y la competencia, lo que dificulta el reconocimiento y la legitimación del desempeño de las masculinidades marginadas e invisibles, como es el caso de las ejercidas por hombres con trastornos mentales.

A pesar de este riesgo, tanto el diario de campo como las entrevistas nos permitieron verificar la positividad de la práctica del fútbol como una actividad de autoafirmación y prestigio que permite el ejercicio de una masculinidad posible, en un contexto en el que estos jóvenes fueron inicialmente excluidos y/o marginados. Por lo tanto, existe un potencial terapéutico al incluir las masculinidades marginadas en las prácticas inherentes a las masculinidades hegemónicas.

A pesar de esta potencialidad, las discusiones sobre patrones de género en los servicios investigados están ausentes y no se abordan los impactos de las demandas e ideales de los modelos hegemónicos de masculinidad en la salud mental de los hombres. Todos los artículos que abordaron la práctica del fútbol en el ámbito de los CAPS, utilizados en este estudio ${ }^{(16,18,19,20)}$, no discutieron los temas relacionados con el género y las masculinidades involucradas en estas actividades.

Se puede afirmar, además, que los servicios no dan visibilidad a las transformaciones en el ejercicio de la masculinidad resultantes de la experiencia del trastorno mental. Incluso en el CAPS-AD, donde la mayoría de los usuarios son hombres, no se identificaron discusiones sobre lo que es o debería ser "masculino" en ninguna actividad observada. Esto corrobora los hallazgos de Zane$1 \mathrm{IO}^{(10)}$, quien señala que las prácticas de salud mental no solo desconocen los problemas de género involucrados en la experiencia del sufrimiento mental, sino que también reifican las desigualdades de género invisibles a los ojos de los servicios.

\section{CONSIDERACIONES FINALES}

Los hallazgos de este estudio muestran el potencial del taller terapéutico de fútbol en la rehabilitación psicosocial de hombres usuarios de los CAPS, partiendo de la reinserción social y cultural en una actividad de gran importancia en el proceso de construcción de la masculinidad y de lo que representa ser hombre en Brasil. Esto realimenta el ejercicio de una masculinidad posible, ya que estos pacientes son marginados en relación con el modelo de masculinidad hegemónica debido a la vivencia del sufrimiento mental.

Se recomiendan acciones complementarias al taller terapéutico de fútbol, en las que 
después de la práctica del fútbol se discuta la importancia de este deporte en la constitución de la subjetividad de los usuarios masculinos y cómo experimentan el ejercicio de sus masculinidades, buscando una reflexión sobre qué es ser hombre. Esta reflexión no se observó en la práctica del servicio investigado y tampoco en la bibliografía científica estudiada para la producción de este artículo.

Los resultados muestran la importancia de los CAPS como espacio para el proceso de reinvención de la sociabilidad de los pacientes con trastornos mentales y señalan la necesidad de crear entornos receptivos para los hombres, que estén relacionados con los procesos de subjetivación masculina y puedan servir como dispositivos de reflexión sobre los patrones de masculinidad, cuestionando cómo la masculinidad hegemónica interfiere, y qué efectos tiene sobre la salud mental y el bienestar de los hombres.

\section{REFERENCIAS BIBLIOGRÁFICAS}

1. Figueiredo WS. Masculinidades e cuidado: diversidade e necessidades de saúde dos homens na atenção primária [Tese de Doutorado]. São Paulo: Faculdade de Medicina, Universidade de São Paulo; 2008.

2. Nascimento MAF. Desaprendendo o silêncio: Uma experiência de trabalho com grupos de homens autores de violência contra a mulher. [Dissertação de Mestrado]. Rio de Janeiro: Universidade do Estado do Rio de Janeiro; 2001.

3. Albuquerque FP, Santos Barros CR, Schraiber LB. Violência e sofrimento mental em homens na atenção primaria a saúde. Revista de Saúde Pública, 2013;47(3):531-539.

4. Schraiber LB, Gomes R, Couto MT. Homens na pauta da saúde coletiva. Ciência \& Saúde Coletiva. 2005;10(1):7-17

5. Couto MT, Schraiber LB. Homens, Saúde e Violência: Novas questões de gênero no campo da Saúde Coletiva. En: Minayo MCS, Coimbra Junior CEA (Org.). Críticas e atuantes: Ciências sociais e humanas em saúde na América Latina. Rio de Janeiro: Fiocruz; 2005. p. 687-706.

6. Schraiber LB, Figueiredo WS, Gomes R, Couto MT, Pinheiro TF, Machin R, Silva GSN, Valença O. Necessidades de saúde e masculinidades: atenção primária no cuidado aos homens. Cadernos de Saúde Pública. 2010;26(5):961-970.

7. Couto MT, Pinheiro TF, Valença O, Machin R, Silva GSN, Gomes R, Schraiber LB, Figueiredo VS. O homem na atenção primária à saúde: discutindo (in)visibilidade a partir da perspectiva de gênero. Interface - Comunicação, Saúde, Educação. 2010;14(33):257-270.
8. Gomes R, Nascimento EF, Araújo FC. Por que os homens buscam menos os serviços de saúde do que as mulheres?: As explicações de homens com baixa escolaridade e homens com ensino superior. Cadernos de Saúde Pública. 2007;23(3):565-574.

9. Campos IO, Ramalho WM, Zanello V. Mental and gender health: The sociodemographic profile of patients in psychosocial attention center. Estudos de Psicologia (Natal). 2017;22(1):68-77.

10. Zanello V, Fiuza G. Saúde mental e gênero: facetas gendradas do sofrimento psíquico. Fractal, Revista de Psicologia. 2015;27(3):238-246.

11. Amarante P. Saúde mental e atenção psicossocial. Rio de Janeiro: Editora Fiocruz; 2007.

12. Granja EMS. Gênero, masculinidades e drogas: trilhas, obstáculos e atalhos nos caminhos para atenção integral aos homens jovens na saúde. [Tese de Doutorado]. Rio de Janeiro: Instituto Nacional de Saúde da Mulher, da Criança e do Adolescente Fernandes Figueira; 2015.

13. Moraes MM. Hombres, masculinidades y atención sanitaria en Brasil: una mirada de genero sobre políticas públicas de reducción de daños. [Tesis de Doctorado]. Barcelona: Universitat Autònoma de Barcelona; 2012.

14. Brasil, Ministério da Saúde. Portaria No. 854, de 22 de Agosto de 2012 [Internet]. 2012 [citado 10 ene 2019]. Disponible en: https://tinyurl.com/ tulwyaj.

15. Pádua FHP, Morais MLS. Oficinas expressivas: Uma inclusão de singularidades. Psicologia USP. 2010;21(2):457-478.

16. Adib LT, Fraga AB, Wachs F, Alves CTP. The body practices concerning mental health: A football workshop capabilities and possibilities in a Psycho- 
social Care Centre. Pensar a Prática, Goiânia. 2010;13(2):1-14.

17. Azevedo EB, Ferreira Filha MO. Práticas inclusivas na rede de atenção à saúde mental: entre dificuldades e facilidades. Ciência \& Saúde. 2012;5(2):60-70.

18. Biffi D, Nasi C. Concepção dos usuários sobre as atividades terapêuticas desenvolvidas em um CAPS AD III. Journal of Nursing UFPE On Line. 2015;9(12):1057-1062.

19. Wachs F, Fraga AB. Educação física em centros de atenção psicossocial. Revista Brasileira de Ciências do Esporte. 2009;31(1):93-107.

20. Furtado RP, Azevedo MC, Nevesa RLR, Vieira PS. O trabalho do professor de educação física nos Caps de Goiânia: identificando as oficinas terapêuticas. Revista Brasileira de Ciências do Esporte. 2018;40(4):353-360.

21. Santos WTM. Modelos de masculinidade na percepção de jovens homens de baixa renda. Barbarói. 2007;(27):130-157.

22. Souza MA. Gênero e raça: a nação construída pelo futebol brasileiro. Cadernos Pagu. 1996;(67):109-152.

23. Denzin NK, Lincoln YS. Introduction: the discipline and practice of qualitative research. En: Denzin NK, Lincoln YS, (eds.). Handbook of qualitative research. London: Sage Publications; 2000. p. 1-29.

24. Deslandes SF, Gomes R. A pesquisa qualitativa em serviços de saúde: notas teóricas. En: Bosi MLM, Mercado FJ, (orgs.). Pesquisa qualitativa de serviços de saúde. Petrópolis: Editora Vozes; 2004. p. 99-120.
25. Zanello V. Saúde mental, gênero e dispositivos: cultura e processos de subjetivação. Curitiba: Appris; 2018.

26. Figueiredo WS, Schraiber LB. Concepções de gênero de homens usuários e profissionais de saúde de serviços de atenção primária e os possíveis impactos na saúde da população masculina, São Paulo, Brasil. Ciência \& Saúde Coletiva. 2011;16(Suppl 1);935-944.

27. Thiollent MJM. Crítica metodológica, investigação social e enquete operária. São Paulo: Polis; 1982.

28. Wielewicki VHG. A pesquisa etnográfica como construção discursiva. Acta Scientiarum, Maringá. 2001;23(1):27-32.

29. Maragno L, Goldbaum M, Gianini RJ, Novaes HMD, César CLG. Prevalência de transtornos mentais comuns em populações atendidas pelo Programa Saúde da Família (QUALIS) no Município de São Paulo, Brasil. Cadernos de Saúde Pública. 2006;22(8):1639-1648.

30. Figueiredo WS. Assistência a saúde dos homens: um desafio para os serviços de atenção primária. Ciência \& Saúde Coletiva. 2005;10(1):105-109.

31. Arilha M, Ridenti SG, Unbehaum MB (org.). Homens e masculinidades: outras palavras. São Paulo: Ecos, Editora 34; 1998.

32. Martinhago F, Oliveira WF. (Des)institucionalização: a percepção dos profissionais dos centros de atenção psicossocial de Santa Catarina, Brasil. Saúde e Sociedade. 2015;24(4):1273-1284.

33. Gastaldo E. "O complô da torcida": futebol e performance masculina em bares. Horizontes Antropológicos. 2005;11(24):107-123.

\section{FORMA DE CITAR}

Albuquerque FP, Schraiber LB. Masculinidad y fútbol: cuestiones de género en una experiencia de rehabilitación psicosocial de hombres en el Distrito Federal, Brasil. Salud Colectiva. 2020;16:e2247. doi: 10.18294/sc.2020.2247.

Recibido: 29-03-2019 | Versión final: 22-11-2019 | Aprobado: 20-12-2019 | Publicado en línea: 17-02-2020.

Esta obra está bajo una licencia de Creative Commons Reconocimiento-NoComercial 4.0 Internacional. Reconocimiento - Permite copiar, distribuir y comunicar públicamente la obra. A cambio, se debe reconocer y citar al autor original. No Comercial - Esta obra no puede ser utilizada con finalidades comerciales, a menos que se obtenga el permiso. 